\title{
Strong Proximal Continuity and Convergence
}

\author{
Agata Caserta, ${ }^{1}$ Roberto Lucchetti, ${ }^{2}$ and Som Naimpally ${ }^{3}$ \\ ${ }^{1}$ Department of Mathematics, Seconda Università degli Studi di Napoli, 81100 Caserta, Italy \\ ${ }^{2}$ Department of Mathematics, Politecnico di Milano, 20133 Milano, Italy \\ ${ }^{3} 96$ Dewson Street, Toronto, ON, Canada M3J 1 P3 \\ Correspondence should be addressed to Roberto Lucchetti; roberto.lucchetti@polimi.it
}

Received 16 October 2012; Accepted 9 January 2013

Academic Editor: Yuriy Rogovchenko

Copyright (C) 2013 Agata Caserta et al. This is an open access article distributed under the Creative Commons Attribution License, which permits unrestricted use, distribution, and reproduction in any medium, provided the original work is properly cited.

\begin{abstract}
In several situations the notion of uniform continuity can be strengthened to strong uniform continuity to produce interesting properties, especially in constrained problems. The same happens in the setting of proximity spaces. While a parallel theory for uniform and strong uniform convergence was recently developed, and a notion of proximal convergence is present in the literature, the notion of strong proximal convergence was never considered. In this paper, we propose several possible convergence notions, and we provide complete comparisons among these concepts and the notion of strong uniform convergence in uniform spaces. It is also shown that in particularly meaningful classes of functions these notions are equivalent and can be considered as natural definitions of strong proximal convergence. Finally we consider a function acting between two proximity spaces and we connect its continuity/strong continuity to convergence in the respective hyperspaces of a natural functor associated to the function itself.
\end{abstract}

\section{Introduction}

In topology and analysis the concepts of uniform continuity and uniform convergence on compacta play a central role. It is well known that a continuous function restricted to a compact set is uniformly continuous on that set. In the setting of metric spaces, Beer and Levi [1] observed that a continuous function is actually uniformly continuous on a sufficiently small enlargement of every compact set. This led them to introduce the concept of strong uniform continuity on a set that is not necessarily compact. The concepts of uniform continuity and strong uniform continuity agree on the whole space, but they may differ on a subset. This phenomenon is interesting since it is rare; generally one comes across concepts which differ globally and agree locally. It is also known that uniform convergence preserves uniform continuity but on the other hand this may fail for strong uniform continuity. Thus in Beer and Levi [1] and Caserta et al. [2] a definition of strong uniform convergence is considered, enjoying the property of preserving strong uniform continuity. This concept is related to the sticking convergence defined by Bouleau [3] (see also [4]). In Beer [5], the author extends the concept of strong uniform continuity to the more general setting of Hausdorff uniform spaces and develops the rudiments of the theory. Since in any uniform space a natural proximity is associated to the uniformity, it is then useful to consider notions of continuity and convergence directly connected to the proximity structure. Thus a notion of proximal continuity has been proposed, with the property of preserving the nearness of two sets. This is a stronger notion than simple continuity, which preserves nearness of a point and a set. The equality of the two properties provides a powerful tool in studying the problem of extension of continuous functions from dense subspaces; see Gagrat and Naimpally [6]. In analogy with the uniform case, a natural concept of convergence is defined in this setting. It is called proximal convergence, or also Leader convergence, since it was defined by Leader [7]. In his investigation Leader in particular showed that uniform convergence implies proximal convergence and that the converse in general does not hold. However, when considering functions acting between the two proximity spaces $(X, \alpha)$ and $(Y, \beta)$, it is known that two convergence modes are equal in the following three cases: (a) $Y$ is totally bounded, (b) the converging net is a sequence or the directed set of the net is linearly ordered, and (c) $X$ is compact (see [7-10]). Furthermore, proximal convergence 
preserves continuity as well as proximal continuity. Then Beer and Levi [1] introduced some new concepts of local-type proximal continuity and local-type proximal convergence, in the setting of metric spaces, and provided some characterizations of strong uniform continuity and strong uniform convergence in terms of the analogous proximal properties. These characterizations rely on convergence of various types on bornologies. Some extensions are also provided in Beer [5].

In this paper, after revising the various concepts of continuity in uniform and proximal spaces, we introduce several forms of strong proximal convergences, and we investigate their connections in the setting of Tychonoff spaces with compatible uniformities and proximities. We also compare them with uniform convergences, and we study them on bornologies. Then we connect uniform and proximal continuity and convergences of functions with the behavior of a natural functor in the hyperspace associated to a given function $f$, that is, the functor $f^{\wedge}(A)=f(A)$. In the hyperspace we consider the proximal topology and the Hausdorff-Bourbaki uniformity. Our results generalize similar ones in Beer and Levi [1] and Di Maio et al. [11-13].

\section{Preliminaries}

Given a topological space $(X, \tau)$, we will denote by $P(X)$ $(C L(X))$ the set of all subsets (closed subsets) of $X$, and by $P_{0}(X)\left(C L_{0}(X)\right)$ the set of all nonempty (nonempty closed) subsets. A central role in the paper will be played by the notion of bornology. Thus, let us start with the following definition.

Definition 1. Given a set $X$, a bornology $\mathscr{B}$ on $X$ is a family of subsets of $X$ which is a covering, closed under finite union, and hereditary. By a base $\mathscr{B}_{0}$ for a bornology $\mathscr{B}$ one means a subfamily of $\mathscr{B}$ that is cofinal with respect to inclusion.

There is the smallest bornology on $X$, denoted by $\mathscr{B}_{f}$, containing (only) the finite subsets of $X$. Of course $P_{0}(X)$ is the greatest bornology on $X$. Another important bornology is the bornology $\mathscr{K}$ of the relatively compact subsets of $X$. In the sequel we shall constantly assume that bornologies have a closed base. We introduce now some relevant properties of bornologies, introduced and studied in $[5,14,15]$.

Definition 2. A bornology $\mathscr{B}$ on a Hausdorff uniform space $(X, \mathscr{U})$ is said to be stable under small enlargements if it contains an enlargement $U(A)$ of each of its members $A ; \mathscr{B}$ is said to be shielded from closed sets if for every $A \in \mathscr{B}$ there is $B \in \mathscr{B}$ such that $A \subset B$ and each neighborhood of $B$ contains an enlargement of $A$.

Clearly each bornology $\mathscr{B}$ that is stable under small enlargements is shielded from closed sets, but the converse does not hold: $\mathscr{B}_{f}$ is always shielded but stable under small enlargements if and only if all points are isolated, and $\mathscr{K}$ is stable under small enlargement if and only if $X$ is locally compact.
In the paper we focus only on Tychonoff topological spaces with associated Efremovič symmetric uniformities. For a complete reference on proximity spaces see Naimpally and Warrack [16]. It is well known that to any uniformity $\mathscr{W}$ on a uniform space $Z$ a natural proximity $\zeta$ is associated: $A \zeta B$ if there is no a symmetric $W \in \mathscr{W}$ such that $W(A) \cap B=$ $\emptyset$. In the other way around the situation is different: given a proximity space $(Z, \alpha)$, there are in general several, not equivalent uniformities on $Z$, having $\alpha$ as a natural proximity. Thus, we shall consider $(X, \mathscr{U}),(Y, \mathscr{V})$ uniform spaces when we deal with uniform structures, and the associated proximity spaces $(X, \alpha),(Y, \beta)$, when we speak about proximal properties. We shall use indifferently the notation $A \underline{\alpha} B$ and $A \ll_{\alpha} B^{c}$ to indicate that $A \alpha B$ is false. We now introduce some continuity notions we shall use throughout the paper.

Definition 3. Let $(X, \mathscr{U}),(Y, \mathscr{V})$ be uniform spaces, and $\mathscr{B}$ a bornology on $X$. One says that a function $f: X \rightarrow Y$ is uniformly continuous if for every $V \subset \mathscr{V}$ there exists $U \subset \mathcal{U}$ such that for every $x, y \in X$ :

$$
y \in U(x) \Longrightarrow f(y) \in V(f(x)) .
$$

Given a nonempty subset $A$ of $X, f$ is uniformly continuous on $A$ if its restriction $\left.f\right|_{A}:(A, \mathscr{U}) \rightarrow(Y, \mathscr{V})$ is uniformly continuous. Finally, we say that $f$ is uniformly continuous on $\mathscr{B}$ if $f$ is uniformly continuous on $A$, for every $A \in \mathscr{B}$.

Remark 4. Uniform continuity can be expressed in the following equivalent form:

$$
E \subset A \Longrightarrow f(U(E) \cap A) \subset V(f(E)) \text {. }
$$

This last remark suggests the following enforcement of uniform continuity.

Definition 5. Let $(X, \mathcal{U}),(Y, \mathscr{V})$ be uniform spaces, and $\mathscr{B}$ a bornology on $X$. let $A$ be a nonempty subset of $X$. One says that the function $f: X \rightarrow Y$ is strongly uniformly continuous on $A$ if for every $V \in \mathscr{V}$ there exists $U \in \mathcal{U}$ such that

$$
E \subset A \Longrightarrow f(U(E)) \subset V(f(E)) \text {. }
$$

We say that $f$ is strongly uniformly continuous on $\mathscr{B}$ if it is strongly uniformly continuous on $A$, for every $A \in \mathscr{B}$.

Definition 6 . Let $(X, \alpha)$ and $(Y, \beta)$ be proximity spaces, and $\mathscr{B}$ a bornology on $X$. We say that the function $f: X \rightarrow Y$ is proximally continuous on $X$ if

$$
\forall E, S \subset X, \quad E \alpha S \Longrightarrow f(E) \beta f(S)
$$

Given a nonempty subset $A$ of $X, f$ is proximally continuous on $A$ if its restriction $\left.f\right|_{A}:(A, \alpha) \rightarrow(Y, \beta)$ is proximally continuous. Finally, we say that $f$ is proximally continuous on $\mathscr{B}$ if $f$ is proximally continuous on $A$, for every $A \in \mathscr{B}$.

Definition 7. Let $(X, \alpha)$ and $(Y, \beta)$ be proximity spaces; let $A$ be a nonempty subset of $X$ and $\mathscr{B}$ a bornology on $X$. One says 
that the function $f:(X, \alpha) \rightarrow(Y, \beta)$ is strongly proximally continuous on $A$ if

$$
\forall E \subset A, \forall S \subset X, \quad E \alpha S \Longrightarrow f(E) \beta f(S) .
$$

Finally, one says that $f$ is strongly proximally continuous on $\mathscr{B}$ if $f$ is strongly proximally continuous on $A$, for every $A \in$ $\mathscr{B}$.

We shall use the notation $C_{\mathscr{B}}^{\mathrm{sp}}(X, Y)$ to denote the family of the functions from $X$ to $Y$ which are strongly proximally continuous on the bornology $\mathscr{B}$.

Remark 8. Proximal and strong proximal continuity on a set can be equivalently expressed in the following way: $f$ is proximally continuous on $A$ if

$$
\forall E \subset A, \forall T \subset f(A), \quad f(E) \ll_{\beta} T \Longrightarrow E \ll_{\alpha} f^{-1}(T) .
$$

$f$ is strongly proximally continuous on $A$ if

$$
\forall E \subset A, \forall T \subset Y, \quad f(E) \ll_{\beta} T \Longrightarrow E \ll_{\alpha} f^{-1}(T) .
$$

We now recall some connections among the introduced continuity notions. Strong uniform continuity on a set implies uniform continuity on that set. The converse does not hold in general; for a complete characterization of equivalence among the two continuities in uniform spaces, see Beer [5]; strong proximal continuity implies proximal continuity, and in general the two notions do not coincide; furthermore (strong) uniform continuity implies (strong) proximal continuity, for the natural proximity associated to the uniformity. On the contrary a proximally continuous function needs not to be uniformly continuous for the uniformities compatible with the proximity. As an example, take a proximity space $(X, \alpha)$ and uniformities $\mathscr{U}_{2}$ and $\mathscr{U}_{1}$ on $X$ such that $\mathscr{U}_{1}$ is strictly finer than $\mathscr{U}_{2}$. The identity map Id $:\left(X, \mathscr{U}_{2}\right) \rightarrow\left(X, \mathscr{U}_{1}\right)$ is not uniformly continuous, but the associated map from the proximal space $(X, \alpha)$ into itself is so. However, if $X, Y$ are metric spaces and $f$ is proximally continuous with respect to the induced proximities, then it is also uniformly continuous with respect to their underlying uniformities (see [17, Corollary 4.4.2]). More generally, it is clear that on the whole bornology $\mathscr{B}_{f}$ the two notions of strong continuity do coincide and are equivalent to continuity of $f$.

For the coincidence of the two notions of uniform continuity and strong uniform continuity on a bornology, let us recall the result proved in Beer [5, Theorem 3.5] that a continuous function acting between two uniform spaces, if it is uniformly continuous on a bornology shielded from closed sets, is automatically strongly uniformly continuous on the bornology. To provide a similar result in the proximity setting, we rephrase the notion of bornology stable under small enlargements, given in Beer [5, Definition 3.2] in a uniform setting, in order to have the analogous property in proximal spaces.

Definition 9. Given a proximity space $(X, \alpha)$ and a bornology $\mathscr{B}$ on it, one says that $\mathscr{B}$ is stable under small enlargements if for every $A$ in $\mathscr{B}$ there is $\mathscr{B}$ in $\mathscr{B}$ such that $A \ll_{\alpha} B$.
The next Lemma is useful to prove when proximal continuity and strong proximal continuity coincide on a bornology.

Lemma 10. Let $(X, \alpha)$ and $(Y, \beta)$ be proximity spaces. Let $A, C \subset X$ such that $A \ll_{\alpha} C$. Suppose that for each $F \subset C$ such that $A \alpha F$ it holds $f(A) \beta f(F)$. Then $f$ is strongly proximally continuous on $A$.

Proof. Take $G \subset X$ such that $A \alpha G$. Let $F=C \cap G$. We claim that $A \alpha F$. Otherwise, setting $H=G \cap C^{c}$, it is $A \alpha H$, but then $G=F \cup H$ is such that $A \underline{\alpha} G$, against the assumption. Thus $A \alpha F$ and then by assumption $f(A) \beta f(F)$. Since $F \subset G$, it follows that $f(A) \beta f(G)$.

Proposition 11. Let $(X, \alpha)$ and $(Y, \beta)$ be two proximity spaces. Let $\mathscr{B}$ be a bornology on $X$ stable under small enlargements. Then the following are equivalent:

(1) $f$ is proximally continuous on $\mathscr{B}$;

(2) $f$ is strongly proximally continuous on $\mathscr{B}$.

Proof. We only need to prove that (1) implies (2). Fix $A \in \mathscr{B}$ and let $C \in \mathscr{B}$ be such that $A \ll_{\alpha} C$. From Lemma 10 it is enough to prove that if $F \subset C$ is such that $A \alpha F$, then $f(A) \beta f(F)$. But this is an immediate consequence of the fact that $f$ is proximally continuous on $C$.

Since the bornology $\mathscr{B}_{f}$ is not stable under small enlargements, but it is shielded from closed sets, it is easily seen that in the above proposition the assumption on the bornology of being stable under small enlargements cannot be weakened, since any function is proximally continuous on $\mathscr{B}_{f}$, but only the continuous functions are strongly proximally continuous on $\mathscr{B}_{f}$.

In Beer [5, Theorem 3.5] a result similar to Proposition 11 in uniform setting is proved.

\section{Convergences}

In this section we recall some notions of uniform and proximal convergence, and related properties. Some of the definitions are classical; some other are new. We also make several comparisons among them.

We first recall the well-known definition of uniform convergence between uniform spaces.

Definition 12. Let $(X, \mathscr{U}),(Y, \mathscr{V})$ be uniform spaces, and let $\mathscr{B}$ be a bornology on $X$. Let $D$ be a directed set and $f_{n}, f \in Y^{X}$, $n \in D$. One says that the net $\left\{f_{n}\right\}$ uniformly converges to $f$ on $A \in \mathscr{B}$ if

$$
\forall V \in \mathscr{V} \exists N: \forall n>N \quad x \in A \Longrightarrow f_{n}(x) \in V(f(x)) .
$$

$\left\{f_{n}\right\}$ uniformly converges to $f$ on $\mathscr{B}$ if it converges uniformly on every $A \in \mathscr{B}$.

Definition 13 (see Beer [5]). Let $(X, \mathscr{U}),(Y, \mathscr{V})$ be uniform spaces, and let $\mathscr{B}$ be a bornology on $X$. Let $D$ be a directed 
set and $f_{n}, f \in Y^{X}, n \in D$. One says that the net $\left\{f_{n}\right\}$ strongly uniformly converges to $f$ on $A$ if

$$
\begin{gathered}
\forall V \in \mathscr{V} \exists N: \forall n>N, \\
\exists U_{n} \in \mathscr{U}: x \in U_{n}(A) \Longrightarrow f_{n}(x) \in V(f(x)) .
\end{gathered}
$$

$\left\{f_{n}\right\}$ strongly uniformly converges to $f$ on $\mathscr{B}$ if it converges strongly uniformly on every $A \in \mathscr{B}$.

Remark 14. Strong uniform convergence on $A$ can be equivalently expressed in the following way:

$$
\begin{gathered}
\forall V \in \mathscr{V} \exists N: \forall n>N, \\
\exists U_{n} \in \mathscr{U}: \forall C \subset A, \\
f_{n}\left(U_{n}(C)\right) \subset V(f(C)) .
\end{gathered}
$$

Evidently for each Hausdorff uniform codomain the strong uniform convergence is finer than the uniform convergence on $Y^{X}$; they collapse on $Y^{X}$ if and only if the bornology $\mathscr{B}$ is stable under small enlargements, Beer [5, Theorem 3.1]; moreover they coincide on $C(X, Y)$ if and only if the bornology $\mathscr{B}$ is shielded from closed sets, Beer [5, Theorem 3.4].

Definition 15 (see Leader [7]). Let $(X, \alpha)$ and $(Y, \beta)$ be proximity spaces, and $\mathscr{B}$ a bornology on $X$. Let $D$ be a directed set and $f, f_{n} \in Y^{X}, n \in D$. The net $\left\{f_{n}\right\}$ proximally converges to $f$ on $A \in \mathscr{B}$ if

$$
\forall C \subset A, \forall T \subset Y: f(C) \ll_{\beta} T, \quad \exists N: \forall n>N \quad f_{n}(C) \ll_{\beta} T .
$$

The net $\left\{f_{n}\right\}$ proximally converges on $\mathscr{B}$ if it proximally converges on $A$ for every $A \in \mathscr{B}$.

We introduce now the following definitions, in order to investigate strong proximal convergence on bornology.

Definition 16. Let $(X, \alpha)$ and $(Y, \beta)$ be proximity spaces, and $\mathscr{B}$ a bornology on $X$. Let $D$ be a directed set and let $f, f_{n} \in$ $Y^{X}, n \in D$. The net $\left\{f_{n}\right\}$ outer proximally converges to $f$ on $A \in \mathscr{B}$ if

$$
\begin{gathered}
\forall C \subset A, \forall T \subset Y: \exists B \in \tau_{X}: C \subset B \wedge f(B) \ll_{\beta} T, \\
\exists N: \forall n>N \exists B_{n} \in \tau_{X}: C \subset B_{n} \subset B \wedge f_{n}\left(B_{n}\right) \ll_{\beta} T .
\end{gathered}
$$

The net $\left\{f_{n}\right\}$ outer proximally converges on $\mathscr{B}$ if it outer proximally converges to $f$ on $A$, for every $A \in \mathscr{B}$.

Definition 17. Let $(X, \tau)$ be a topological space, let $(Y, \beta)$ be a proximity space, and let $\mathscr{B}$ be a bornology on $X$. Let $D$ be a directed set and let $f, f_{n} \in Y^{X}, n \in D$. One says that $\left(\left\{f_{n}\right\}, f\right)$ satisfies property $(\nabla)$ on $A \in \mathscr{B}$ if

$$
\begin{gathered}
\forall C \subset A, \forall T \subset Y: f(C) \ll_{\beta} T, \\
\exists N: \forall n>N \exists B_{n} \in \tau_{X}: C \subset B_{n} \wedge f_{n}\left(B_{n}\right) \ll_{\beta} T .
\end{gathered}
$$

One says that $\left(\left\{f_{n}\right\}, f\right)$ satisfies property $(\nabla)$ on $\mathscr{B}$ if it satisfies property $(\varnothing)$ on every $A \in \mathscr{B}$.
Definition 18. Let $(X, \alpha)$ and $(Y, \beta)$ be proximity spaces, and let $\mathscr{B}$ be a bornology on $X$. Let $D$ be a directed set and let $f, f_{n} \in Y^{X}, n \in D$. One says that $\left(\left\{f_{n}\right\}, f\right)$ satisfies property (\$) on $A \subset X$ if

$$
\begin{gathered}
\forall C \subset A, \forall T \subset Y: \exists B: C \ll_{\alpha} B \wedge f(B) \ll_{\beta} T, \\
\exists N: \forall n>N \exists B_{n}: C \ll_{\alpha} B_{n} \subset B \wedge f_{n}\left(B_{n}\right) \ll_{\beta} T .
\end{gathered}
$$

One says that $\left(\left\{f_{n}\right\}, f\right)$ satisfies property (中) on $\mathscr{B}$ if it satisfies property (\$) on every $A \in \mathscr{B}$.

We observe at first that there is an equivalent way, often used in the sequel, to express the above definitions. For instance, outer proximal convergence of $\left\{f_{n}\right\}$ to $f$ on $\mathscr{B}$ can be equivalently expressed in the following way: let $(X, \alpha)$ and $(Y, \beta)$ be proximity spaces, and $\mathscr{B}$ a bornology on $X$. Let $D$ be a directed set and $f, f_{n} \in Y^{X}, n \in D$. The net $\left\{f_{n}\right\}$ outer proximally converges to $f$ on $\mathscr{B}$ if

$$
\begin{gathered}
\forall A \in \mathscr{B}, \forall T \subset Y: \exists B \in \tau_{X}: A \subset B \wedge f(B) \ll_{\beta} T, \\
\exists N: \forall n>N \exists B_{n} \in \tau_{X}: A \subset B_{n} \subset B \wedge f_{n}\left(B_{n}\right) \ll_{\beta} T .
\end{gathered}
$$

Remark 19. In the case the bornology $\mathscr{B}$ is the bornology of the finite subsets of $X$, proximal convergence coincides with pointwise convergence. This is no longer true for outer proximal convergence, as Example 23 shows. However it is clearly true in the case the limit function $f$ is continuous, but in such a case a more general result holds (see Proposition 25).

Remark 20. Property $(\nabla)$ usually does not define topological convergence, at least on the whole space $Y^{X}$. For instance, it can happen that $\left(\left\{f_{n}:=f\right\}, f\right)$ does not fulfill property $(\nabla)$. Moreover, the next Example 21 shows that on the whole space $Y^{X}$ the (\$) limit of a net does not need to be unique. However both conditions are interesting and natural properties to consider and become topological convergences on important subsets of $Y^{X}$. In particular, $(\nabla)$ is a sticking convergence type, as defined in Bouleau [3] and Bouleau [4], while (\$) looks like a natural type of proximal convergence, since it directly appeals to proximity properties and not to topological properties of the two proximity spaces.

In the sequel, when dealing with metric spaces, we always intend that the proximity is the natural proximity associated with the metric.

Example 21. In this example we see some pathological phenomenon associated to properties $\boldsymbol{\alpha}$ and $\varnothing$, from the point of view of convergence of nets. Let $X=\mathbb{R}^{2}, Y=\mathbb{R}$, and $\mathscr{B}$ the bornology of the bounded sets. Let

$$
f(x, y)= \begin{cases}0 & \text { if } y=0 \\ 1 & \text { otherwise. }\end{cases}
$$

Then $\left(\left\{f_{n}=f\right\}, f\right)$ does not satisfy property $(\nabla)$. Let $X=\mathbb{R}$, $Y=[0,+\infty]$. Let $f_{n}: X \rightarrow \mathbb{R}$ be the zero function, for every $n$. Then observe that $\left(\left\{f_{n}\right\}, f\right)$ satisfies (\&) for any $f$ valued zero on a dense subset of $\mathbb{R}$. 
Coming back to the relations between the above properties/convergences, we immediately have the following.

Proposition 22. Let $(X, \alpha)$ and $(Y, \beta)$ be proximity spaces, and $\mathscr{B}$ a bornology on $X$. Let $D$ be a directed set and $f, f_{n} \in Y^{X}$, $n \in D$.

(1) If $\left(\left\{f_{n}\right\}, f\right)$ satisfies $(\nabla)$ on a set $A$, then $f_{n}$ outer proximally converges on the set $A$, whence the same holds on a bornology.

(2) If $\left(\left\{f_{n}\right\}, f\right)$ satisfies $(\nabla)$ on a set $A$, then $f_{n}$ proximally converges on the set $A$, whence the same holds on a bornology.

Example 23. In this example we show that implication (1) in Proposition 22 is not an equivalence. Let $X=Y=\mathbb{R}$ and $\mathscr{B}=\mathscr{B}_{f}$. Define

$$
\begin{aligned}
& f(x)= \begin{cases}0 & \text { if } x=0 \\
\frac{1}{x} & \text { otherwise }\end{cases} \\
& f_{n}(x)= \begin{cases}n & \text { if } x=0 \\
\frac{1}{x} & \text { otherwise }\end{cases}
\end{aligned}
$$

Then $\left\{f_{n}\right\}$ outer proximal converges to $f$, but does not proximal converge on $\mathscr{B}_{f}$; thus in particular $\odot$ does not hold.

On the other hand, on $Y^{X}$ proximal and outer convergence are independent, as the following example shows.

Example 24. In light of Example 23, we only need to produce a sequence proximally converging, but not outer proximally converging on $\mathscr{B}$. Let $X=Y=\mathbb{R}$ let $\mathscr{B}=\mathscr{B}_{f}$ and $f$ the zero function. Let $\alpha_{1}, \alpha_{2}, \ldots, \alpha_{k}, \ldots$ be an enumeration of the rational numbers and set

$$
f_{n}(x)= \begin{cases}\frac{k}{n} & \text { if } x=\alpha_{k} \\ 1 & \text { otherwise. }\end{cases}
$$

Taking any open set $B$ the set $T=[-\varepsilon, \varepsilon]$ fulfills $f(B) \ll_{\beta} T$, while for any $B \sup _{x \in B} f_{n}(B)=+\infty$, and thus there is no outer proximal convergence. Observe that the limit function $f$ is continuous, while the approximating functions are not.

With some (weak) assumptions on the functions $f_{n}, f$ further relations hold.

Proposition 25. Let $(X, \alpha)$ and $(Y, \beta)$ be proximity spaces, and $\mathscr{B}$ a bornology on $X$. Let $D$ be a directed set and $f, f_{n} \in Y^{X}$, $n \in D$.

(1) If $f \in C(X, Y)$ and $f_{n} \rightarrow f$ outer proximally on $A \in$ $\mathscr{B}$, then $f_{n} \rightarrow f$ proximally on $A \in \mathscr{B}$, whence the same holds on $\mathscr{B}$;
(2) If $f \in C(X, Y)$ and $f_{n} \rightarrow f$ outer proximally on $A \epsilon$ $\mathscr{B}$, then $\left(\left\{f_{n}\right\}, f\right)$ satisfies $(\nabla)$ on $A \in \mathscr{B}$, whence the same holds on $\mathscr{B}$;

(3) If $f_{n} \in C(X, Y)$ and if $f_{n} \rightarrow f$ proximally on $A \in \mathscr{B}$, then $f_{n} \rightarrow f$ outer proximally on $A \in \mathscr{B}$, whence the same holds on $\mathscr{B}$.

Proof. (1) Let $A \in \mathscr{B}$ and let $T \subset Y$ be such that $f(A) \ll_{\beta} T$. There is $E \in \tau_{Y}$ such that $f(A) \ll_{\beta} E \ll_{\beta} T$. Let $\tau_{X} \ni B:=$ $f^{-1}(E)$. Thus $f(B) \ll_{\beta} T$. By assumption there is $N$ such that there are $B_{n} \in \tau_{X}$ such that $A \subset B_{n} \subset B$ and $f_{n}\left(B_{n}\right) \ll_{\beta} T$, and thus for all $n \geq N f_{n}(A) \ll_{\beta} T$.

(2) Suppose $f(A) \ll_{\beta} T$. Then there is $O \in \tau_{Y}$ such that $f(A) \ll_{\beta} O \ll_{\beta} T$. We conclude by applying the definition of outer proximal convergence to $B:=f^{-1}(O)$.

(3) Let $A \in \mathscr{B}$ and let $T \subset Y$ be such that there is $B \in \tau_{X}$ with $A \subset B$ and $f(B) \ll_{\beta} T$. By assumption there is $N$ such that for all $n \geq N, f_{n}(B) \ll_{\beta} T$. Thus there are for all $n$ open sets $W_{n}$ such that $f_{n}(B) \ll_{\beta} W_{n} \ll_{\beta} T$. Let $\tau_{X} \ni B_{n}:=f^{-1}\left(W_{n}\right) \cap B$. Hence $A \subset B_{n} \subset B$ and $f_{n}\left(B_{n}\right) \ll_{\beta} T$.

The following Corollary is immediate.

Corollary 26. Let $(X, \alpha)$ and $(Y, \beta)$ be proximity spaces. Then in the space $C(X, Y)$ the following are equivalent:

(1) $f_{n} \rightarrow f$ for the proximal convergence;

(2) $f_{n} \rightarrow f$ for the outer proximal convergence;

(3) property $(\nabla)$ holds for $\left(\left\{f_{n}\right\}, f\right)$.

Thus in the class of continuous functions proximal convergence is a sticking type convergence described also by means of property $(\nabla)$.

The next Lemma is useful to characterize outer proximal convergence.

Lemma 27. Let $(X, \alpha),(Y, \beta)$ be two proximity spaces, $\mathscr{B}$ a bornology on $X$ let $f \in Y^{X}$ and $A \in \mathscr{B}$. Then the following are equivalent:

(1) $f$ is strongly proximally continuous on $A$;

(2) for every $T \subset Y$ such that $f(A) \ll_{\beta} T$,

$$
\exists B: A \ll_{\alpha} B \wedge f(B) \ll_{\beta} T .
$$

Proof. Let $T$ be such that $f(A) \ll_{\beta} T$. Then by (2) there is $B$ such that $A \ll_{\alpha} B$ and $f(B) \ll_{\beta} T$. Thus $A \ll_{\alpha} B \subset f^{-1}(T)$ and so (2) implies (1). Conversely, let $T$ be such that $f(A) \ll_{\beta} T$. Then there is $S$ such that $f(A) \ll_{\beta} S \ll_{\beta} T$. Then by (1) $A \ll_{\alpha} f^{-1}(S)$, and there is $B$ such that $A \ll_{\alpha} B \ll_{\alpha} f^{-1}(S)$; it follows that $f(B) \subset S \ll_{\beta} T$.

Observe that in the above condition the set $B$ can be equivalently supposed to be open.

From Lemma 27 we immediately get the following corollary. 
Corollary 28. Let $(X, \alpha)$ and $(Y, \beta)$ be proximity spaces and $\mathscr{B}$ a bornology on $X$. Then in the class $C_{\mathscr{B}}^{s p}(X, Y)$ a net $\left\{f_{n}\right\}$ proximally converges if and only if property (\$) holds.

Proof. This is an immediate consequence of Lemma 27.

We now introduce two new properties on a pair $\left(\left\{f_{n}\right\}, f\right)$, where as usual $n \in D$, with $D$ a directed set. Similar to the case of property $(\nabla)$, they do not define a topological convergence on $Y^{X}$, but on important subclasses of $Y^{X}$ they do.

Definition 29 . Let $(X, \alpha)$ and $(Y, \beta)$ be proximity spaces. Let $f: X \rightarrow Y$ and $\mathscr{B}$ be a bornology on $X$. One says that $\left(\left\{f_{n}\right\}, f\right)$ satisfies property $(\diamond)$ on $A \in \mathscr{B}$ if

$$
\begin{gathered}
\forall T \subset Y: f(A) \ll_{\beta} T \exists B \subset X: \\
A \ll_{\alpha} B \wedge \exists N: \forall n>N f_{n}(B) \ll_{\beta} T .
\end{gathered}
$$

One says that $\left(\left\{f_{n}\right\}, f\right)$ satisfies property $(\diamond)$ on $\mathscr{B}$ if it satisfies property $(\diamond)$ on each $A \in \mathscr{B}$.

The next example shows that a constant net does not need to fulfill the property $(\diamond)$. Thus the same considerations made for property $(\nabla)$ in Remark 20 apply to $(\diamond)$ as well. Naturally, we shall call property $(\diamond)$ equiproximal convergence, when it is a topological convergence.

Example 30. Let $X$ be an infinite dimensional separable Hilbert space with orthonormal basis $\left\{e_{n}: n \in \mathbb{N}\right\}$ (e.g., $l^{2}$ with $\left.e_{n}=(0,0, \ldots, 1, \ldots, \ldots)\right)$. Let $f: X \rightarrow[0,+\infty)$ be the following function:

$$
f(x)=\sum_{n=0}^{\infty}\left(x, e_{n}\right)^{2 n} .
$$

Setting $A=B(0 ; 1)$, the unit ball in the Hilbert space, happens that $f(A)=[0,1]$, while for any enlargement $H$ of $A f(H)=$ $[0, \infty)$. Thus one more time the constant net $\left\{f_{n}=f\right\}$ does not converge to $f$.

The second property is meaningful instead only when a bornology is specified. For this reason we do not make comparisons of this property with the other ones with specific examples on sets.

To prove our results, we start by giving a new definition, in the context of proximity spaces: it is the natural adaptation in this setting of the definition given by Beer in [5] in the uniform case.

Definition 31. Let $(X, \alpha)$ be a proximity space and $\mathscr{B}$ a bornology on it. One says that $\mathscr{B}$ is shielded from closed sets if for each $A \in \mathscr{B}$ there is $B \in \mathscr{B}$ such that each open set $O$ containing $B$ is such that $A \underline{\alpha} O^{c}$.

In the sequel we shall naturally say, for sets $A$ and $B$ as above, that $B$ is a shield for $A$.

Definition 32. Let $(X, \tau)$ be a topological space, let $(Y, \beta)$ be a proximity space, and let $\mathscr{B}$ be a bornology on $X$. Let $D$ be a directed set and let $f, f_{n} \in Y^{X}, n \in D$. One says that $\left(\left\{f_{n}\right\}, f\right)$ satisfies property $(\boldsymbol{A})$ on $\mathscr{B}$ if

$$
\begin{gathered}
\forall A \in \mathscr{B} \quad \forall T \subset Y: f(A) \ll_{\beta} T \\
\exists C \in \mathscr{B}: C \text { shields } A \wedge \exists N: \forall n>N \quad f_{n}(C) \ll_{\beta} T .
\end{gathered}
$$

Property $(\diamond)$ is a stronger notion than both property (\$) and proximal convergence. However in the next propositions we see that on bornologies with particular features the new properties coincide with previous convergences.

Theorem 33. Let $(X, \alpha)$ and $(Y, \beta)$ be two proximity spaces; let $D$ be a directed set and $f, f_{n} \in Y^{X}, n \in D$. Let $\mathscr{B}$ be a bornology on $X$ and stable under small enlargements; let $f$ be strongly proximally continuous on $\mathscr{B}$. Then the following are equivalent:

(1) $\left(\left\{f_{n}\right\}, f\right)$ satisfies property (A) on $\mathscr{B}$;

(2) $\left\{f_{n}\right\}$ proximally converges to $f$ on $\mathscr{B}$;

(3) $\left(\left\{f_{n}\right\}, f\right)$ satisfies property $(\diamond)$ on $\mathscr{B}$.

On the other hand, if for every $f \in \mathbb{R}^{X}$ which is strongly proximally continuous on $\mathscr{B},(2)$ and (3) are equivalent on $\mathscr{B}$, then $\mathscr{B}$ is stable under small enlargements.

Proof. It is enough to prove that (2) and (3) are equivalent. Let $A \in \mathscr{B}$ and let us prove that if $f_{n} \rightarrow f$ proximally on $A$ then $\left\{f_{n}\right\}$ satisfies property $(\diamond)$ on $\mathscr{B}$. Thus, suppose $T$ is such that $f(A) \ll_{\beta} T$. Since $f$ is strongly proximally continuous on $A$ and $\mathscr{B}$ is stable under small enlargements, from Lemma 27 we know that there is $B \in \mathscr{B}$ such that $f(B) \ll_{\beta} T$. Since $f_{n} \rightarrow f$ on $B$, then eventually $f_{n}(B) \ll_{\beta} T$, and this concludes the proof of the first part. Let us now prove the second statement. Suppose $\mathscr{B}$ is not stable under small enlargements. We shall produce a net $\left\{f_{n}\right\}$ proximally converging to a strongly proximally continuous limit function $f$ on $\mathscr{B}$, but on the other hand $\left(\left\{f_{n}\right\}, f\right)$ does not satisfy property $(\diamond)$ on $\mathscr{B}$. There is $A \in \mathscr{B}$ such that for every $B \in \mathscr{B}$ it is not the case that $A \ll_{\alpha} B$. Let $D=\{B \in \mathscr{B}: A \subset B\}$, directed by inclusion. Observe that, for every $F \subset X$ such that $A \ll_{\alpha} F$, for every $B \in D$ there is $x_{B F} \in F \backslash B$. Now define

$$
f_{\mathscr{B}}(x)= \begin{cases}1 & \text { if } x=x_{B F} \text { for some } F \\ 0 & \text { otherwise }\end{cases}
$$

We now prove that $f_{B}$ proximally converges on $\mathscr{B}$ to the function $f$ valued zero everywhere, but on the other hand $\left(\left\{f_{B}\right\}, f\right)$ does not satisfy property $(\diamond)$ on $A \in \mathscr{B}$. The first statement is clear, since for all $C \in D$ such that $B \subset C$, it is $f_{C}(x)=0$ on $C$, whence on $A$. About the second setting $T=[-1 / 2,1 / 2]$, it is $f(A) \ll T$, but for any $F \subset X$ such that $A \ll_{\alpha} F$, for every $B \in D, 1 \in f_{B}(F)$, and this ends the proof. 
Theorem 34. Let $(X, \alpha)$ and $(Y, \beta)$ be two proximity spaces let $D$ be a directed set and $f, f_{n} \in Y^{X}, n \in D$. Let $\mathscr{B}$ be a bornology on $X$ shielded from closed sets; let $f$ be strongly proximally continuous on $\mathscr{B}$. Then the following are equivalent:

\section{(1) $\left(\left\{f_{n}\right\}, f\right)$ satisfies property $(\boldsymbol{A})$; \\ (2) $\left\{f_{n}\right\}$ proximally converges to $f$ on $\mathscr{B}$.}

On the other hand, if $X$ is a normal space, if for every $f \in \mathbb{R}^{X}$ which is strongly proximally continuous on $\mathscr{B}$, (1) and (2) are equivalent on $\mathscr{B}$, then $\mathscr{B}$ is shielded from closed sets.

Proof. Obviously we only need to prove that (2) implying (1), since (1) implies (2) is always true, with no assumptions on $f$ and $\mathscr{B}$. Let $\left\{f_{n}\right\}$ be a net proximally converging to $f$ on $\mathscr{B}$, let $f$ be strongly proximally continuous on $\mathscr{B}$, let $A \in$ $\mathscr{B}$, and suppose $f(A) \ll_{\beta} T$. Then there is $M \subset Y$ such that $f(A) \ll_{\beta} M \ll_{\beta} T$. Since $f$ is strongly proximally continuous on $\mathscr{B}$, it is $A \ll_{\alpha} f^{-1}(M)$. Take $B \in \mathscr{B}$ such that $B$ shields $A$. It is easy to see then that $\mathscr{B} \ni C:=B \cap f^{-1}(M)$ shields $A$. Moreover $f(C) \subset M \ll_{\beta} T$. Since $f_{n} \rightarrow f$ proximally on $C \in$ $\mathscr{B}$, then eventually $f_{n}(C) \ll_{\beta} T$, and this concludes the proof of the first part. Suppose now $\mathscr{B}$ is not shielded from closed sets. We shall produce a net $\left\{f_{n}\right\}$ proximally converging to a strongly proximally continuous limit function $f$ on $\mathscr{B}$, but on the other hand $\left(\left\{f_{n}\right\}, f\right)$ does not satisfy property $(\boldsymbol{A})$ on $\mathscr{B}$. Thus, there is $A \in \mathscr{B}$ such that for every $B \in \mathscr{B}$ there exists a closed set $F_{B}$ with $F_{B} \cap B=\emptyset$ and $A \alpha F_{B}$. Let $D=\{B \in \mathscr{B}$ : $A \subset B \wedge B$ is closed $\}$, directed by inclusion. Now, for every $B \in D$ find a continuous function $f_{B}$ which is valued 0 on $B$ and 1 on $F$. Mimicking the proof of Theorem 34, it is easy to see that $f_{B}$ proximally converges to $f$ on $\mathscr{B}$ (since $\mathscr{B}$ has a closed base), but $\left(\left\{f_{B}\right\}, f\right)$ does not satisfy property $(\boldsymbol{A})$ on $A \in \mathscr{B}$.

As a conclusion, observe that on $C(X, Y)$ convergences proximal, outer proximal and property $(\nabla)$ coincide, and in the subset $C_{\mathscr{B}}^{\mathrm{sp}}(X, Y)$ of $C(X, Y)$ of the strongly proximal continuous functions also (\$) is equivalent to proximal convergence. In the next example we see that (\$) can be in general different from other convergences in $C(X, Y) \backslash$ $C_{\mathscr{B}}^{\mathrm{sp}}(X, Y)$.

Example 35. Let $X$ be the following subset of $\mathbb{R}^{2}$ :

$$
X=\{(x, y): x \geq 1, y \geq 0\}
$$

Let $A=\{(x, y) \in X: y=0\}$. Consider the bornology $\mathscr{B}$ of the sets of the form $S \cup\left\{x_{1}\right\} \cup \cdots \cup\left\{x_{k}\right\}$, with $k \in \mathbb{N}$ and $S \subset A$. Finally, consider $f_{n}(x, y)=(1 / n) x y, f(x, y)=0$. Then $f_{n} \rightarrow f$ proximally, but $\left(\left\{f_{n}\right\}, f\right)$ does not satisfy property (中). If instead we take $f(x, y)=x y, f_{n}(x, y)=x y+(1 / n) x^{2}$, we see that $f_{n}$ does not proximally converge on $A$, while (\$) holds on $\mathscr{B}$.

From the previous considerations the following corollary is immediate.
Corollary 36. Let $(X, \alpha)$ and $(Y, \beta)$ be two proximity spaces let $\mathscr{B}$ be a bornology on $X$ stable under small enlargements. Then in the space $C_{\mathscr{B}}^{s p}(X, Y)$ the following are equivalent:

(1) $f_{n} \rightarrow f$ for the proximal convergence;

(2) $f_{n} \rightarrow f$ for the outer proximal convergence;

(3) property $(\nabla)$ holds for $\left(\left\{f_{n}\right\}, f\right)$ (sticking convergence);

(4) property $(\diamond)$ holds for $\left(\left\{f_{n}\right\}, f\right)$ (equiproximal convergence);

(5) property (\$) holds for $\left(\left\{f_{n}\right\}, f\right)$;

(6) property (A) holds for $\left(\left\{f_{n}\right\}, f\right)$.

We end the section showing some connections among strong uniform convergence and the proximal convergences introduced before.

Proposition 37. Let $(X, \mathcal{U}),(Y, \mathscr{V})$ be uniform spaces, with associated proximities $\alpha, \beta$; let $\mathscr{B}$ be a bornology on $X$. Let $\left\{f_{n}\right\}$, $n \in D, D$ a directed set, be a net strongly uniformly converging to $f$ on $\mathscr{B}$. Then $\left(\left\{f_{n}\right\}, f\right)$ satisfies property (\$) on $\mathscr{B}$.

Proof. Fix $A \in \mathscr{B}$ and let $T \subset Y$ be such that there is $B$ such that $B \ll_{\alpha} A$ and $f(A) \ll_{\beta} T$; hence there is $V$ such that $V(f(A)) \subset T$. Let $W$ be such that $W^{2} \subset V$. Since $f_{n}$ strongly uniformly converges to $f$ on $A$, there exists $N$ such that for all $n>N$ there are $U_{n}$ such that for all $x \in U_{n}(A)$, $f_{n}(x) \subset V(f(x))$. Let $U_{1}$ such that $U_{1}(A) \subset B$. There is $U_{n}^{\prime} \subset U_{1} \cap U_{n}$ and there is $\widehat{U}_{n}$ be such that $\widehat{U}_{n}^{2} \subset U_{n}^{\prime}$. Thus $A \subset U_{n}^{\prime}(A)=B_{n} \subset B$. Since $\widehat{U}_{n}(A) \subset \widehat{U}_{n}^{2}(A) \subset B_{n}$, it follows that $A \ll_{\alpha} B_{n} \subset B$. Moreover, for all $x \in \mathscr{B}_{n}, W\left(f_{n}(x)\right) \subset$ $W^{2}(f(x)) \subset V(f(x)) \subset T$, that is, $f_{n}\left(B_{n}\right) \ll_{\beta} T$. result.

The next proposition provides a similar, yet independent,

Proposition 38. Let $(X, \mathcal{U}),(Y, \mathscr{V})$ be uniform spaces, with associated proximities $\alpha, \beta$ let $\mathscr{B}$ be a bornology on $X$. Let $\left\{f_{n}\right\}$ be a net strongly uniformly converging to $f$ on $\mathscr{B}$. Then $\left\{f_{n}\right\}$ outer proximal converges to $f$ on $\mathscr{B}$.

Proof. Fix $B \in \mathscr{B}$ and let $T \subset Y$ be such that there is $A \in \tau_{X}$ with $B \subset A$ and $f(A) \ll_{\beta} T$; thus there is $V$ such that $V(f(A)) \subset T$. Let $W$ be such that $W^{2} \subset V$. Since $f_{n}$ strongly uniformly converges to $f$ on $B$, there exists $N$ such that for all $n>N$ there are $U_{n}$ such that for all $x \in U_{n}(B)$, $f_{n}(x) \in V(f(x))$. Let $B \subset\left(U_{n}(B) \cap A\right)=S_{n} \subset A$. Then all $S_{n}$ are open in $X$ and for all $x \in S_{n}, W\left(f_{n}(x)\right) \subset W^{2}(f(x)) \subset$ $V(f(x)) \subset T$, that is, $f\left(S_{n}\right) \ll_{\beta} T$.

\section{Convergence on Hyperspaces}

In this section we show that the notions of strong uniform continuity (convergence) and strong proximal continuity (convergence) restricted to a bornology are equivalent to continuity (convergences) on hyperspaces with suitable topologies. 
Given a function $f:(X, \tau) \rightarrow(Y, \lambda)$ acting from a topological space to another topological space, it is natural to consider the two following functors associated to $f$ and acting on hyperspaces:

$$
f^{\wedge}: P_{0}(X) \longrightarrow P_{0}(Y), \quad f^{*}: C L(X) \longrightarrow C L(Y),
$$

defined as

$$
f^{\wedge}(A)=f(A), \quad f^{*}(A)=\overline{f(A)} .
$$

We remember that by hyperspace the set $P_{0}(X)$ (or a subset of it, like $C L(X))$ is intended, endowed with some topology making continuous the embedding $x \rightarrow\{x\}$ of $X$ in $P_{0}(X)$. In particular, in this section we shall deal with a net of functions $\left\{f_{n}\right\}, f_{n}:(X, \alpha) \rightarrow(Y, \beta)$ and a function $f$, and we want to connect convergence on a bornology, in various senses, of $f_{n}$ to $f$, with convergence of the associated nets $\left\{f_{n}^{\wedge}\right\}$ and $\left\{f_{n}^{*}\right\}$. Our focus will be on the proximal topology in the hyperspace of a proximal space, and on the Hausdorff Bourbaki uniform topology when considering uniform spaces. As it is well known, these topologies are the join of two topologies, a so-called upper topology and a socalled lower topology.

A basic upper neighborhood of $C \in P_{0}(Y)$, where $(Y, \beta)$ is a proximity space, is

$$
\mathscr{I}:=\left\{H \subset Y: H \ll_{\beta} T\right\},
$$

for some $T \subset Y$ with $C \ll_{\beta} T$, and $T$ is from a given fixed family of subsets of $Y$. Equivalently, a basic neighborhood of $C \subset Y$ is

$$
\mathscr{I}:=\left\{W \in P_{0}(Y): W \underline{\beta} F\right\},
$$

where $F \subset Y$ and $C \beta F$ and $F$ is from a given fixed family of subsets of $Y$. The natural lower topology associated with the former upper one is the lower Vietoris topology (depending only from the topology on $X$, and indicated by $V_{\beta}^{-}$) whose join with the upper Hausdorff topology gives rise to the wellknown proximal topology, indicated from now on by $\sigma_{\beta}$. We remember that a subbase for the lower Vietoris topology is given by $\mathrm{O}^{-}$, where $\mathrm{O}$ is an open set in $Y$ and $\mathrm{O}^{-}$indicates the family of all sets $F \in P_{0}(Y)$ such that $F \cap O \neq \emptyset$. Instead, if $(X, \mathcal{U})$ is a uniform space, in the hyperspace it is possible to consider the so-called Hausdorff-Bourbaki uniformity. As usual, it consists of a lower and an upper part. A typical neighborhood of a set $A$ in the upper uniform topology is the following family of sets:

$$
\left\{B \in P_{0}(X): B \subset U(A)\right\},
$$

where $U \in \mathcal{U}$. Similarly, a typical neighborhood of a set $A$ in the lower uniform topology is the following family of sets:

$$
\left\{B \in P_{0}(X): A \subset U(B)\right\},
$$

where $U \in \mathcal{U}$. It is known that, when considering the proximity naturally associated with the uniformity $\mathcal{U}$, the upper proximal Hausdorff topology agrees with the upper uniform topology (see [18], page 50).

Our first intent is to show that we can limit our analysis to one of the two functors. Our choice will be on $f^{\wedge}$.
Definition 39. Given a topological space $(Z, \tau)$, let $\sigma$ be a topology on the set $P_{0}(Z)$ of the nonempty subsets of $Z$. One shall say that $\sigma$ is a compatible topology on $Z$ if for each $F \in P_{0}(Z)$ and $N \in \sigma, N$ is a neighborhood of $F$ if and only if it is a neighborhood of any set $A$ such that $F \subset A \subset \mathrm{cl} F$.

Proposition 40. Let $(X, \alpha)$ and $(Y, \beta)$ be two proximity spaces, let $f: X \rightarrow Y$ be a function and let $\tau, \sigma$ be compatible topologies on $P_{0}(X)$ and $P_{0}(Y)$, respectively. Let $\mathscr{B}$ be a bornology on $X$ and denote by $\overline{\mathscr{B}}$ the set $\overline{\mathscr{B}}=\{\bar{B}: B \in \mathscr{B}\}$. Then the following are equivalent:
(1) $f^{\wedge}: \mathscr{B} \rightarrow P_{0}(Y)$ is $(\tau, \sigma)$-continuous;
(2) $f^{*}: \overline{\mathscr{B}} \rightarrow C L(Y)$ is $(\tau, \sigma)$-continuous.

It is clear that the hyperspace topologies introduced above are compatible topologies.

Here is our first result.

Proposition 41. Let $(X, \alpha),(Y, \beta)$ be proximity spaces, and let $f:(X, \alpha) \rightarrow(Y, \beta)$ be a given function. The following are equivalent:

(1) $f$ is proximally continuous on $B$;

(2) $f^{\wedge}:\left(P_{0}(B), H_{\alpha}^{+}\right) \rightarrow\left(P_{0}(Y), H_{\beta}^{+}\right)$is continuous;

(3) $f^{\wedge}:\left(P_{0}(B), \sigma_{\alpha}\right) \rightarrow\left(P_{0}(Y), \sigma_{\beta}\right)$ is continuous.

Proof. (1) $\Rightarrow$ (2) Without loss of generality, we can assume $B=X$. Let us prove that, given $A \in P_{0}(X)$, for every neighborhood $L$ of $f^{\wedge}(A)$ there is a neighborhood $N$ of $A$ such that $f^{\wedge}(N) \subset L$. We can assume $L=\left\{T: T \ll_{\beta} M\right\}$, for some $M$ such that $f(A) \ll_{\beta} M$. Thus there is $H$ such that $f(A) \ll_{\beta} H \ll_{\beta} M$. Then $A \ll_{\alpha} f^{-1}(H)$. Take $N=\{C$ : $\left.C \ll_{\alpha} f^{-1}(H)\right\}$. Then for all $C \in N$ it is $f(C) \subset H \ll_{\beta} M$; thus $f^{\wedge}(N) \subset L$.

$(2) \Rightarrow$ (1) By contradiction, there are $A, S$ such that $A \alpha S$ and $f(A) \beta f(S)$. Let $L$ be the following neighborhood of $f^{\wedge}(A): L=\{B: B \beta f(S)\}$. Let $U$ be a neighborhood of $A$ :

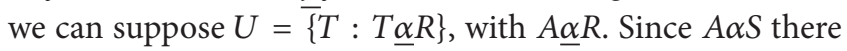
exists $x_{U} \in S \backslash T$. Thus $\left\{x_{U}\right\} \in U$ but $f\left(\left\{x_{U}\right\}\right) \notin L$, so that for no $U$ neighborhood of $A$ in $P_{0}(X)$ it happens that $f^{\wedge}(U) \subset L$, and as a result $f^{\wedge}$ is not continuous at $A$.

$(3) \Leftrightarrow(1)$ It is enough to prove that $f$ proximally continuous implies $f^{\wedge}\left(P_{0}(B), V_{\alpha}^{-}\right) \rightarrow\left(P_{0}(Y), V_{\beta}^{-}\right)$is continuous. But actually continuity of $f$ on $X$ is enough to get it. For, let $O$ be an open set such that $f(A) \cap O \neq \emptyset$. Take $x \in A$ such that $f(x) \in O$. Since $f$ is continuous at $x$, there is an open set $I$ in $X$ such that for all $z \in I f(z) \in O$. Thus $I^{-}$is a neighborhood of $A$ such that, for all $B \in I^{-}, f(B) \in O^{-}$.

The next result instead deals with the same issue, but when the function $f$ is supposed to be strongly proximal continuous, rather than merely proximally continuous. We do not provide the proof, since it mimics that one of Proposition 41. Compare the result of Proposition 42 with Theorem 3.3 in Beer and Levi [1]. 
Proposition 42. Let $(X, \alpha),(Y, \beta)$ be proximity spaces, and let $f:(X, \alpha) \rightarrow(Y, \beta)$ be a given function. The following are equivalent:

(1) $f$ is strongly proximally continuous at every $B \in P_{0}(A)$;

(2) $f^{\wedge}:\left(P_{0}(X), H_{\alpha}^{+}\right) \rightarrow\left(P_{0}(Y), H_{\beta}^{+}\right)$is continuous at every $B \in P_{0}(A)$;

(3) $f^{\wedge}:\left(P_{0}(X), \sigma_{\alpha}\right) \rightarrow\left(P_{0}(Y), \sigma_{\beta}\right)$ is continuous at every $B \in P_{0}(A)$.

We now provide analogous results, in uniform spaces. We shall prove only the second result; the proof of the first one is similar.

Proposition 43. Let $(X, \mathscr{U}),(Y, \mathscr{V})$ be uniform spaces, and let $f:(X, \mathscr{U}) \rightarrow(Y, \mathscr{V})$ be a given function. The following are equivalent:

(1) $f$ is uniformly continuous on $A$;

(2) $f^{\wedge}:\left(P_{0}(A), H_{\mathscr{U}}^{+}\right) \rightarrow\left(P_{0}(Y), H_{\mathscr{V}}^{+}\right)$is continuous;

(3) $f^{\wedge}:\left(P_{0}(A), H_{\mathscr{U}}\right) \rightarrow\left(P_{0}(Y), H_{\mathscr{V}}\right)$ is continuous.

Proposition 44. Let $(X, \mathscr{U}),(Y, \mathscr{V})$ be uniform spaces, and let $f:(X, \mathscr{U}) \rightarrow(Y, \mathscr{V})$ be a given function. The following are equivalent:

(1) $f$ is strongly uniformly continuous on $A$;

(2) $f^{\wedge}:\left(P_{0}(X), H_{\mathscr{U}}^{+}\right) \rightarrow\left(P_{0}(Y), H_{\mathscr{V}}^{+}\right)$is continuous at every $B \in P_{0}(A)$;

(3) $f^{\wedge}:\left(P_{0}(X), H_{\mathscr{U}}\right) \rightarrow\left(P_{0}(Y), H_{\mathscr{V}}\right)$ is continuous at every $B \in P_{0}(A)$.

Proof. (2) $\Rightarrow$ (1) Suppose by contradiction that (1) is not true. Then there is $V \in \mathscr{V}$ such that for all $U \in \mathscr{U}$ there exist $E_{U} \subset$ $A$ and points $x_{U}, y_{U}$, with

$$
x_{U} \in E_{U}, \quad\left(x_{U}, y_{U}\right) \in U \quad\left(f\left(x_{U}\right), f\left(y_{U}\right)\right) \notin V .
$$

Since $f^{\wedge}$ is by assumption continuous with respect to the upper Hausdorff uniformities, given $V \in \mathscr{V}$ as above, there is $U \in \mathcal{U}$ such that $f(U(E) \subset V(f(E))$ for all subsets $E$ of $A$. But this contradicts (1), for $E=\left\{x_{U}\right\}$.

(1) $\Rightarrow$ (3) By contradiction, suppose there is $F \subset A$ such that there is $V \in \mathscr{V}$ such that there exists $G_{U}$ with $F \subset U\left(G_{U}\right)$ and $f(F) \varsubsetneqq V\left(f\left(G_{U}\right)\right)$; that is, there are $x_{U} \in F$ and $y_{U} \in$ $U\left(x_{U}\right)$ with $\left(f\left(x_{U}, f\left(y_{U}\right)\right) \notin V\right.$. But this contradicts strong uniform continuity of $f$ at $A$.

The following corollary generalizes Theorem 2.2. in Di Maio et al. [11], which establishes the same result in metric spaces.

Corollary 45. Let $(X, \mathscr{U}),(Y, \mathscr{V})$ be uniform spaces, and let $f:(X, \mathscr{U}) \rightarrow(Y, \mathscr{V})$ be a given function. The following are equivalent:

(1) $f$ is strongly uniformly continuous on $P_{0}(X)$;

(2) $f$ is uniformly continuous on $X$;
(3) $f^{\wedge}:\left(P_{0}(X), H_{\mathscr{U}}^{+}\right) \rightarrow\left(P_{0}(Y), H_{\mathscr{V}}^{+}\right)$is continuous;

(4) $f^{\wedge}:\left(P_{0}(X), H_{\mathscr{U}}\right) \rightarrow\left(P_{0}(Y), H_{\mathscr{V}}\right)$ is continuous.

The next results instead deal with convergences on a bornology.

Proposition 46. Let $(X, \alpha)$ and $(Y, \beta)$ be two proximity spaces; let $D$ be a directed set and $f, f_{n} \in Y^{X}, n \in D$. Let $\mathscr{B}$ be a bornology on $X$. Then the following are equivalent:

(1) $\left\{f_{n}\right\}$ proximally converges to $f$ on $\mathscr{B}$;

(2) $\left\{f_{n}^{\wedge}\right\}$ pointwise converges to $f^{\wedge}$ at every $A \in \mathscr{B}$.

Proof. Suppose $\left\{f_{n}\right\}$ proximally converges to $f$ on $A \in \mathscr{B}$, and let $\mathscr{I}$ be a neighborhood of $f^{\wedge}(A)$. We suppose $\mathscr{I}=$ $\{B \subset Y: B \beta F\}$, for some $F \subset Y$, such that $f(A) \beta F$. Then $f_{n}(A) \beta F$ eventually, so that $f_{n}^{\wedge}(A) \in \mathscr{I}$ eventually. The opposite implication follows the same pattern.

In the sequel we shall use the following definition of convergence, that we shall call topological convergence.

Definition 47 . Let $(U, \tau)$ and $(V, \sigma)$ be topological spaces. A net $\left\{f_{n}\right\}, n \in D, D$ a directed set, topologically converges to $f$ if for every $u \in U$, for every neighborhood $\mathcal{N}$ of $f(u)$ there is a neighborhood $\mathscr{M}$ of $u$ and $N$ such that for every $n>N f_{n}(\mathscr{M}) \subset \mathcal{N}$.

Proposition 48. Let $(X, \alpha))$ and $(Y, \beta)$ be two proximity spaces; let $D$ be a directed set and $f, f_{n} \in Y^{X}, n \in D$. Let $\mathscr{B}$ be a bornology on $X$. Then the following are equivalent:

(1) $\left\{f_{n}\right\}$ equiproximally converges to $f$ on $\mathscr{B}$;

(2) $\left\{f_{n}^{\wedge}\right\}:\left(P_{0}(X), H_{\alpha}^{+}\right) \rightarrow\left(P_{0}(Y), H_{\beta}^{+}\right)$topologically converges to $f^{\wedge}$ at the members of $\mathscr{B}$;

(3) $\left\{f_{n}^{\wedge}\right\}:\left(P_{0}(X), \sigma_{\alpha}\right) \rightarrow\left(P_{0}(Y), \sigma_{\beta}\right)$ topologically converges to $f^{\wedge}$ at the members of $\mathscr{B}$.

Proof. To show that (1) implies (2), we take $A \in \mathscr{B}$ and a neighborhood $\mathscr{I}$ of $f^{\wedge}(A)$ in $P_{0}(Y)$. Without loss of generality there exists $T \subset Y$ such that $\mathscr{I}=\left\{H \subset Y: H \ll_{\beta} T\right\}$, with $f(A) \ll_{\beta} T$. Then there exist $N$ and $B\left(\in \tau_{X}\right)$ such that for all $n>N f_{n}(B) \ll_{\beta} T$. Thus $\mathscr{B}=:\left\{C \subset X: C \ll_{\alpha} B\right\}$ is a neighborhood of $A$ such that $f_{n}^{\wedge}(\mathscr{B}) \subset \mathscr{I}$, and this shows the claim. For the converse relation, let $A \in \mathscr{B}$ and let $T$ be such that $f(A) \ll_{\beta} T$. Consider the following neighborhood $\mathscr{I}$ of $f^{\wedge}(A)$ :

$$
\mathscr{I}=\left\{H \subset Y: H \ll_{\beta} T\right\} .
$$

From (2) there are $N$ and an open set $O$ such that for all $n>N f_{n}^{\wedge}(O) \in \mathscr{I}$. Since $O$ is open in $P_{0}(Y)$ and contains $A$ there is $L \subset X$ such that $O \supset\left\{R: R \ll_{\alpha} L\right\}$. There is $B \in \tau_{X}$ such that $A \ll_{\alpha} B \ll_{\alpha} L$. Thus $f_{n}(B) \subset f_{n}(O) \subset \mathscr{I}$ and so $f_{n}(B) \ll_{\beta} T$. To conclude the proof, it is enough to show that equiproximal convergence implies convergence of $\left\{f_{n}^{\wedge}\right.$ ) for the lower Vietoris topology, on $P_{0}(X)$ and $P_{0}(Y)$. Thus, take an open set $O$ such that $f(A) \in O^{-}$. Then there is $x \in A$ 
such that $f(x) \in O$. Thus $f(\{x\}) \ll_{\beta} O$, and there exists $B$, that without loss of generality we can suppose to be open, such that $f_{n}(B) \ll_{\beta} O$. This implies that for all $C \in B^{-}, f_{n}(C) \in O^{-}$, and this ends the proof.

Specializing the above results to the bornology $P_{0}(X)$ and collecting some previous statements we can establish the following interesting corollary.

Corollary 49. Let $(X, \alpha)$ and $(Y, \beta)$ be two proximity spaces; let $D$ be a directed set and $f, f_{n} \in Y^{X}, n \in D$. Then the following are equivalent:

(1) $\left\{f_{n}\right\}$ proximally converges to $f$ on $P_{0}(X)$;

(2) $\left\{f_{n}\right\}$ equiproximally converges to $f$ on $P_{0}(X)$;

(3) $\left\{f_{n}^{\wedge}\right\}:\left(P_{0}(X), H_{\alpha}^{+}\right) \rightarrow\left(P_{0}(Y), H_{\beta}^{+}\right)$topologically converges to $f^{\wedge}$;

(4) $\left\{f_{n}^{\wedge}\right\}:\left(P_{0}(X), \sigma_{\alpha}\right) \rightarrow\left(P_{0}(Y), \sigma_{\beta}\right)$ topologically converges to $f^{\wedge}$;

(5) $\left\{f_{n}^{\wedge}\right\}$ pointwise converges to $f^{\wedge}$ at every $A \in P_{0}(X)$.

Proof. We remind of that, as shown in Naimpally [19], continuity with respect to upper Vietoris topology on graphs automatically implies pointwise convergence, and this implies continuity with respect to the lower Vietoris topology: exactly the same proof applies when considering upper proximal topology instead of upper Vietoris topology.

\section{References}

[1] G. Beer and S. Levi, "Strong uniform continuity, Journal of Mathematical Analysis and Applications, vol. 350, no. 2, pp. 568589, 2009.

[2] A. Caserta, G. Di Maio, and L. Holá, "Arzelà's Theorem and strong uniform convergence on bornologies," Journal of Mathematical Analysis and Applications, vol. 371, no. 1, pp. 384-392, 2010.

[3] N. Bouleau, "Une structure uniforme sur un espace F(E; F)," Cahiers de Topologie et Géométrie Différentielle Catégoriques, vol. 11, no. 2, pp. 207-214, 1969.

[4] N. Bouleau, "On the coarsest topology preservingcontinuity," http://arxiv.org/abs/math/0610373.

[5] G. Beer, "The Alexandroff property and the preservation of strong uniform continuity," Applied General Topology, vol. 11, no. 2, pp. 117-133, 2010.

[6] M. S. Gagrat and S. A. Naimpally, "Proximity approach to extension problems," Fundamenta Mathematicae, vol. 71, no. 1, pp. 63-76, 1971.

[7] S. Leader, "On the completion of proximity spaces by local clusters," Fundamenta Mathematicae, vol. 48, pp. 201-215, 1960.

[8] O. Njastad, "Some properties of proximity and generalized uniformity," Mathematica Scandinavica, vol. 12, pp. 47-56, 1963.

[9] A. Di Concilio and S. A. Naimpally, "Proximal convergence," Monatshefte für Mathematik, vol. 103, no. 2, pp. 93-102, 1987.

[10] A. Di Concilio and S. Naimpally, "Proximal set-open topologies," Bollettino della Unione Matematica Italiana B, vol. 3, no. 1, pp. 173-191, 2000.
[11] G. Di Maio, E. Meccariello, and S. Naimpally, "Hypercontinuous convergence in function spaces," Questions and Answers in General Topology, vol. 22, no. 2, pp. 157-162, 2004.

[12] G. Di Maio, E. Meccariello, and S. Naimpally, "Hypercontinuous convergence in function spaces. II," Ricerche di Matematica, vol. 54, no. 1, pp. 245-254, 2005.

[13] G. Di Maio, E. Meccariello, and S. A. Naimpally, "A natural functor for hyperspaces," Topology Proceedings, vol. 29, no. 2, pp. 385-410, 2005.

[14] G. Beer and S. Levi, "Pseudometrizable bornological convergence is Attouch-Wets convergence," Journal of Convex Analysis, vol. 15, no. 2, pp. 439-453, 2008.

[15] G. Beer, C. Costantini, and S. Levi, "Bornological convergence and shields," Mediterranean Journal of Mathematics, pp. 1-32, 2011.

[16] S. Naimpally and B. D. Warrack:, Proximity Spaces, Cambridge University Press, London, UK, 2008.

[17] A. Di Concilio, "Proximity: a powerful tool in extension theory, function spaces, hyperspaces, boolean algebras and point-free geometry," in Beyond Topology, F. Mynard and E. Elliott, Eds., vol. 486 of Contemporary Mathematics, pp. 89-112, American Mathematical Society, Providence, RI, USA, 2009.

[18] S. Naimpally, Proximity Approach to Problems in Topology and Analysis, Oldenbourg, München, Germany, 2009.

[19] S. A. Naimpally, "Graph topology for function spaces," Transactions of the American Mathematical Society, vol. 123, pp. 267$272,1966$. 


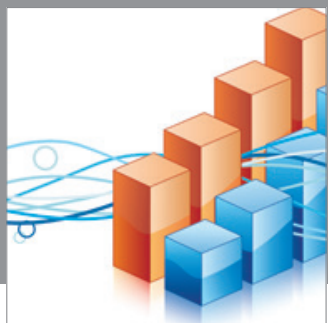

Advances in

Operations Research

mansans

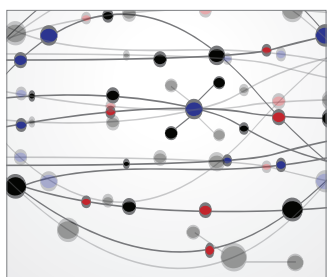

The Scientific World Journal
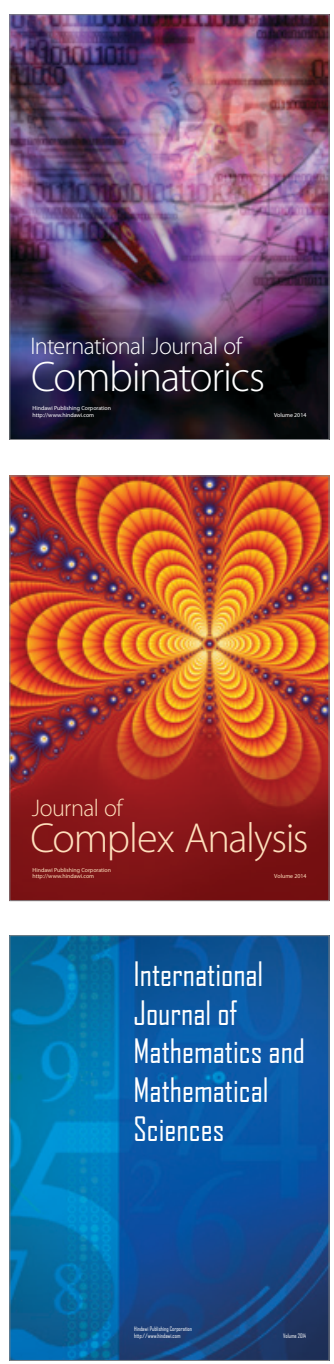
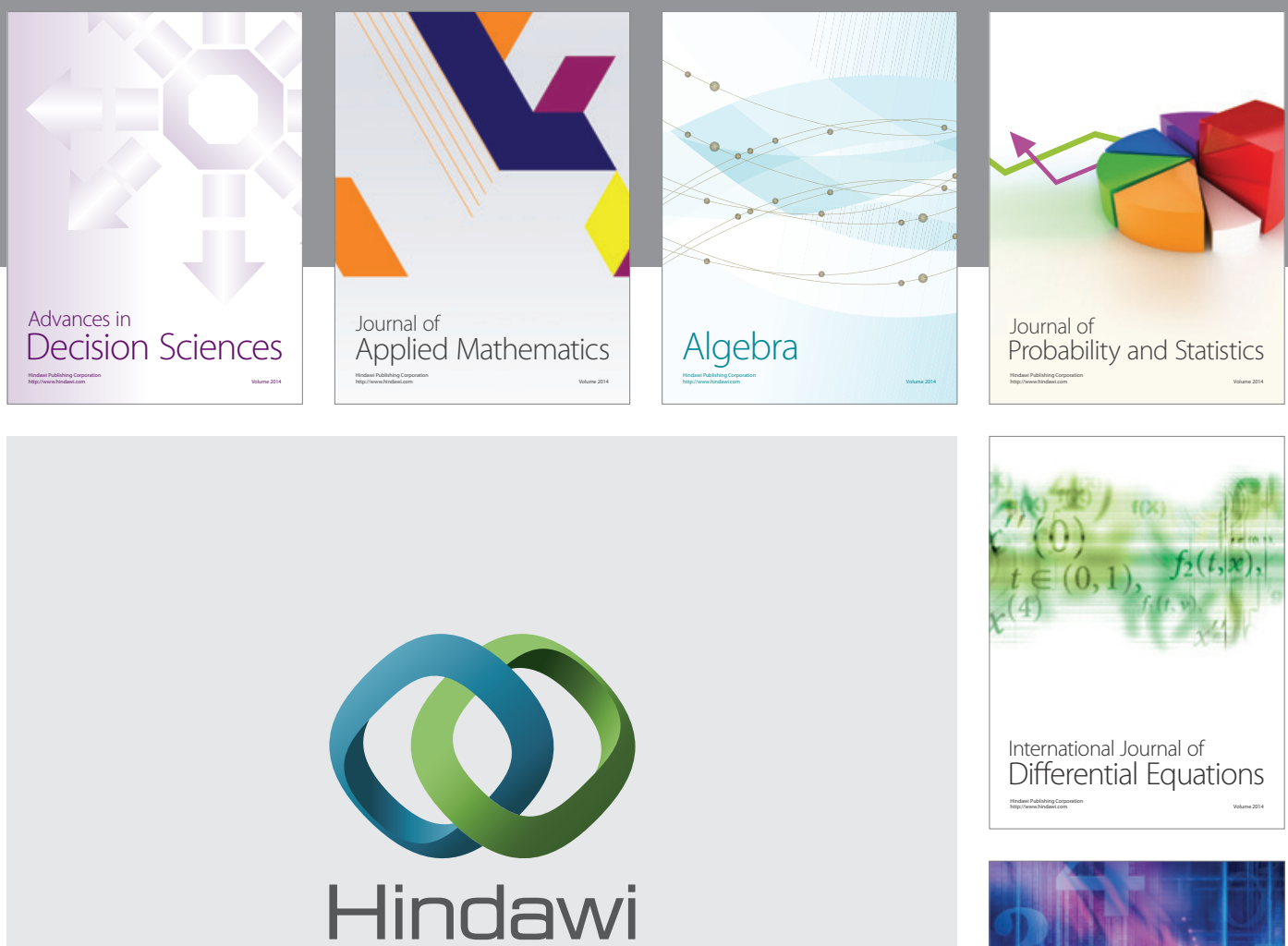

Submit your manuscripts at http://www.hindawi.com
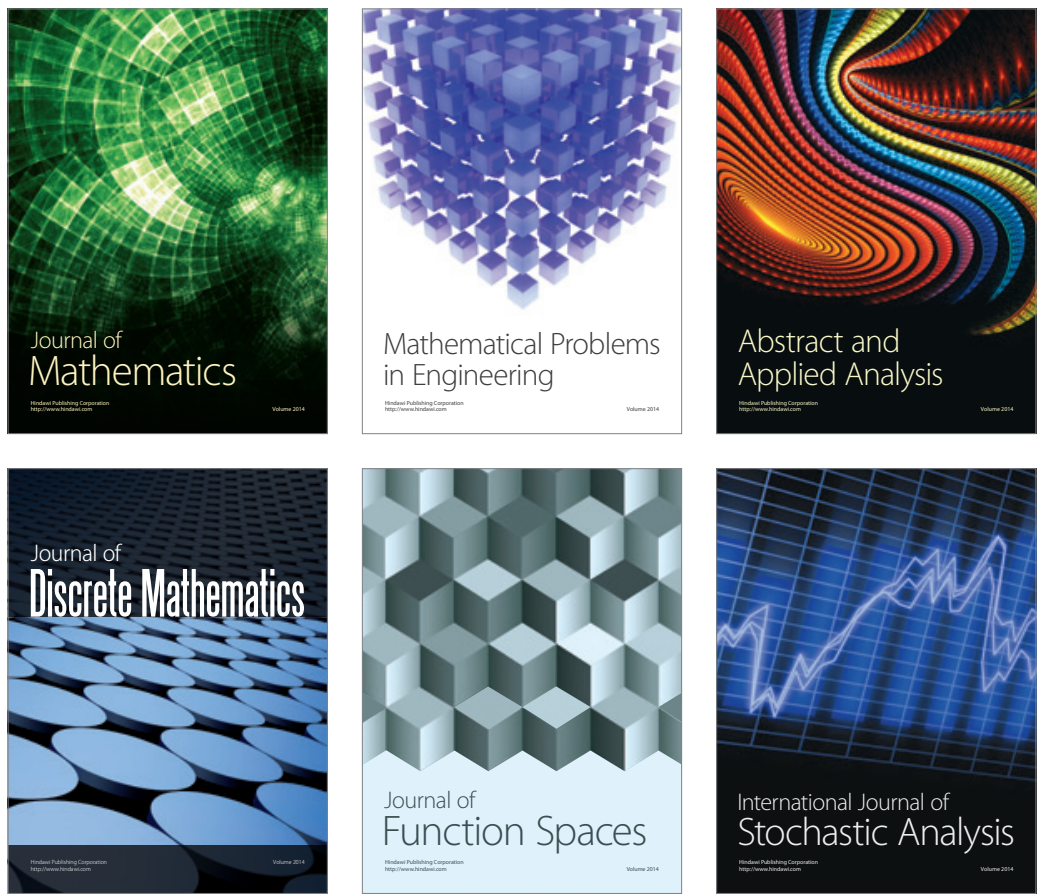

Journal of

Function Spaces

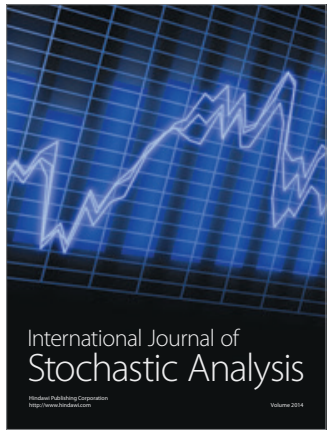

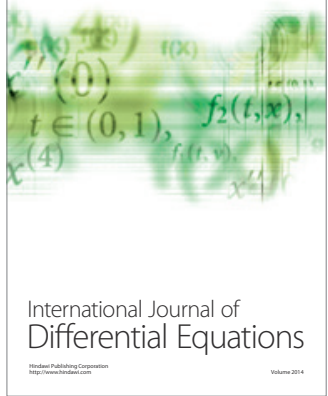
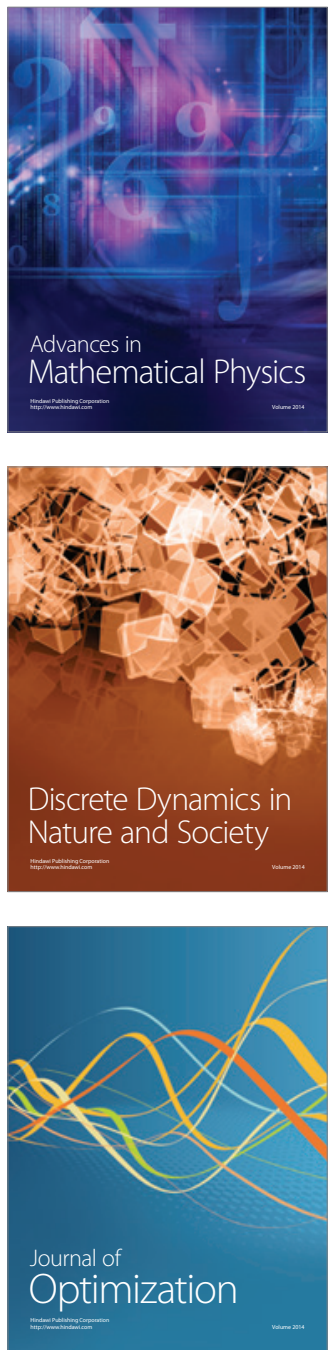